\title{
La tradition ethnographique dans la recherche sur les gangs. Un état de l'art
}

The Ethnographic Tradition in Gang Research

\section{Scott Decker}

Traducteur : Miriam Perier

\section{(2) OpenEdition}

Édition électronique

URL : https://journals.openedition.org/conflits/20194

DOI : 10.4000/conflits.20194

ISSN : $1777-5345$

Éditeur :

CECLS - Centre d'études sur les conflits - Liberté et sécurité, L'Harmattan

\section{Édition imprimée}

Date de publication : 10 octobre 2018

Pagination : $39-58$

ISBN : 978-2-343-16812-8

ISSN : $1157-996 \mathrm{X}$

\section{Référence électronique}

Scott Decker, «La tradition ethnographique dans la recherche sur les gangs. Un état de l'art », Cultures \& Conflits [En ligne], 110-111 | été/automne 2018, mis en ligne le 10 octobre 2020, consulté le 07 janvier 2022. URL : http://journals.openedition.org/conflits/20194; DOl : https://doi.org/10.4000/ conflits.20194 


\section{La tradition ethnographique dans la recherche sur les gangs. Un état de l'art 1}

\section{Scott DECKER}

Scott Decker est professeur fondateur de l'école de criminologie et justice criminelle de l'université d'Arizona. Ses spécialités de recherche sont les gangs, la violence et la délinquance. Il est membre de la Société américaine de criminologie et de l'Académie des sciences de la justice criminelle. Il est l'auteur de dix-buit ouvrages et de plus de 140 articles scientifiques, incluant Life in the Gang: Family, Friends and Violence (Cambridge, 1996), Policing Immigrants: Local Law Enforcement on the Front Lines (University of Chicago, 2016) et Competing for Control: Gangs and the Social Order of Prisons (Cambridge University Press, 2019). Il est membre de l'équipe d'évaluation du programme Proponte' Mas au Honduras.

$\mathrm{I}_{\mathrm{r} e \mathrm{~m}}^{\mathrm{s}}$ est rare qu'une méthodologie soit si fortement associée à un domaine de recherche que l'ethnographie à l'étude des gangs. Les premiers travaux sur ce type de groupes étaient uniquement des ethnographies ${ }^{2}$. L'étude des gangs a pris une part essentielle dans l'évolution de la criminologie, ce qui a rendu possibles à la fois des développements théoriques et l'élaboration de réponses aux crimes. Les travaux de Short et Strodtbeck sur les processus de groupe, de Cohen sur la culture, de Cloward et Ohlin sur le rôle des structures sociales et de Miller sur les valeurs ont tous été fondamentaux, du point de vue théorique, pour la criminologie au sens large, mais aussi pour l'étude des gangs ${ }^{3}$.

1. Traduit de l'anglais par Miriam Perier.

2. Puffer J., The Boy and His Gang, Cambridge, Riverside Press, 1912 ; Thrasher F., The Gang: A Study of 1313 Gangs in Chicago, University of Chicago Press, 1927.

3. Short J., Strodtbeck F., Group Process and Gang Delinquency, Chicago, University of Chicago Press, 1965 ; Cloward R.A., Ohlin L.E., Types of delinquent subcultures, New York, New York School of Social Work of Columbia University, 1958 ; Cohen A., Delinquent Boys: The Culture of the Gang, New York, Free Press, 1955 ; Miller W., « Lower Class Culture as a Generating Milieu of Gang Delinquency », Journal of Social Issues, 14, 1958, pp. 5-19. 
La plupart des nombreuses enquêtes ethnographiques ont été publiées à la fin du XXe siècle et se sont concentrées sur l'étude des groupes criminels dans une ville en particulier. On peut citer les travaux de Moore sur Los Angeles, de Hagedorn sur Milwaukee, de Sanders sur San Diego et de Decker et Van Winkle sur Saint-Louis ${ }^{4}$. D'autres recherches ont suivi, qui sont allées au-delà des lieux consacrés de l'étude des gangs américains qu'ont pu être Chicago, Los Angeles et la Rust Belt ${ }^{5}$. La méthode ethnographique reste préconisée pour la collecte de données.

Peu d'études ont été menées en Europe avant la fin du siècle dernier, sans doute parce que les gangs ne s'y sont développés que tardivement. Dans ce paysage, l'ouvrage de James Patrick, A Glasgow Gang Observed fait figure d'exception et deviendra, plus tard, un classique ${ }^{6}$. Cette recherche documente les effets des structures sociales et de la culture du couteau sur l'émergence et les activités des gangs de rue dans l'une des villes les plus dangereuses du Royaume-Uni. Au regard de ce qui s'est fait aux États-Unis, les travaux ethnographiques sur les gangs européens révèlent une implication plus profonde des chercheurs par rapport aux sujets et aux sites analysés. L'un des exemples les plus remarquables de ce type de travaux est la longue étude menée en Allemagne et coordonnée par Bucerius sur des immigrés venant d'États islamiques ${ }^{7}$.

L'une des questions fondamentales qui émerge dans toute analyse transculturelle ou comparative sur les gangs est celle de l'hétérogénéité/homogénéité. Les gangs diffèrent-ils en fonction des pays et des cultures, reflétant ainsi des traditions locales, des histoires et des modèles de relations hétérogènes ? Ou bien les gangs ont-ils une nature invariable ? Leur caractère fondamental transcende-t-il la culture, les structures et les relations ? La question de la culture est centrale dans notre article et par cette notion, nous entendons les valeurs et les croyances partagées, incarnées dans des symboles, des comportements et des rituels. L'un des objectifs principaux de notre contribution à ce numéro de la revue Cultures $\mathcal{E}$ Conflits, est par ailleurs de documenter l'état de l'ethnographie des gangs.

4. Moore J., Homeboys: Gangs, Drugs, and Prison in the Barrios of Los Angeles, Philadelphia, Temple University Press, 1978 ; Moore J., Going Down to the Barrio: Homeboys and Homegirls in Change, Philadelphia, Temple University Press, 1991 ; Hagedorn J., People and Folks: Gangs, Crime, and the Underclass in a Rustbelt City, Chicago, Lake View, 1988 ; Sanders W., Drive-Bys and Gang Bangs: Gangs and Grounded Culture, Chicago, Aldine, 1993 ; Decker S., Van Winkle B., Life in the Gang: Family, Friends and Violence, New York, Cambridge University Press, 1996.

5. La Rust Belt désigne les villes industrielles de la «ceinture de la rouille » au centre-est des États-Unis parmi lesquelles : Chicago, Detroit, Cleveland, Pittsburg. Ces villes industrielles ont été fortement affectées par le développement technologique.

6. Patrick J., A Glasgow Gang Observed, Londres, Methuen, 1973.

7. Bucerius S., "What Else Should I Do? Cultural Influences on the Drug Trade on Migrants in Germany ", Journal of Drug Issue, 37, 2007, pp. 673-697. 
À la suite des travaux de Decker et Pyrooz ${ }^{8}$, nous présenterons et distinguerons quatre périodes distinctes de l'ethnographie des gangs. La première période, dite classique, regroupe les études ethnographiques sur les groupes criminels menées avant 1970. Puis, la deuxième période, qui s'étend de 1970 à la fin du XXe siècle, est largement dominée par les études nord-américaines consacrées à une seule ville. La troisième période est celle de l'expansion européenne, qui inclut un ensemble de travaux qu'on peut relier à la plateforme Eurogang Research Platform ${ }^{9}$. Enfin, la quatrième période, qualifiée de "Rodgers Revolution" (révolution Rodgers) concerne les enquêtes ethnographiques sur les gangs menées dans les Caraïbes, en Amérique Centrale et du Sud, et qui ont été influencées en partie par les travaux et la personnalité de Dennis Rodgers.

Nous considérons comme ethnographiques les recherches qui s'appuient sur une observation directe, des entretiens, et sur l'utilisation de termes et de catégories natifs ${ }^{10}$. La structure de notre article suivra ces quatre développements de l'ethnographie. Soulignons également ici la forte incompatibilité entre des méthodes qualitatives et quantitatives dans l'étude sur les gangs, tant pour les questions posées que pour les conclusions.

L'utilisation de méthodes ethnographiques est très fortement liée à la recherche historique et contemporaine sur les gangs, en particulier aux premières heures de la recherche sur ce sujet dans un pays, qu'il s'agisse de pays d'Amérique du Nord, d'Europe, ou d'Amérique centrale ou du Sud. La recherche ethnographique sur les groupes criminels s'est concentrée sur des questions de processus, en particulier les processus de groupes ainsi que les dynamiques de changement dans l'évolution des gangs et de leurs membres. La plupart de ces recherches emploient une approche "émique ", c'est-à-dire qu'elles mettent l'accent sur le sens des activités, des relations et des symboles du point de vue des individus, des membres.

Si nous retenons quatre périodes dans cet article, des travaux antérieurs avaient plutôt fait ressortir trois époques distinctes de l'ethnographie des gangs : la classique, l'interstitielle et la contemporaine ${ }^{11}$. La recherche nordaméricaine a dominé les deux premières périodes tandis que la recherche européenne (y compris celle menée au Royaume-Uni) a dominé la troisième ère. Cette recherche européenne se distingue par l'importance qu'elle accorde au

8. Decker S., Pyrooz D., " Gang Violence: Context, Culture, and Country », in McDonald G. (ed.), Small Arms Survey, Londres, Oxford University Press, 2010 ; Decker S., Pyrooz D., " Gangs as Organized Crime Groups », in Decker S., Pryooz D. (eds.), Handbook of Gangs, Maiden, John Wiley \& Sons, 2011.

9. Maxson C. L., Finn-Aage E., Gang transitions and transformations in an International Context, Switzerland, Springer, 2016.

10. Emerson R., Contemporary Field Research, Boston, Little, Brown, 1983.

11. Decker S., Pyrooz D., op. cit., 2010. 
rôle de la culture, de l'ethnicité et des activités non criminelles des membres des groupes observés. Cela reflète les caractéristiques des nouveaux groupes d'immigrés ainsi que les aspects structurels des villes, où la notion de territoire (appelé turf, en anglais) est moins partagée par les membres des gangs.

\section{Les ethnographies de gang classiques}

La plupart des travaux de cette période sont d'ordre descriptif et font partie d'études sociologiques plus larges. Généralement, ces recherches se sont surtout intéressées aux questions d'immigration, d'urbanisation, de pauvreté et d'ethnicité et il s'agit là de questions centrales et constantes de la criminologie et de l'étude de la délinquance. Un certain nombre de ces ethnographies descriptives se sont intéressées à l'exclusion sociale des membres de gangs, entrainant des situations de discrimination tant ethniques qu'économiques. Lewis, par exemple, a observé les conflits ethniques opposant les nouveaux groupes d'immigrants dans la ville de New York ${ }^{12}$. En effet, la question ethnique est au cour de la formation des gangs et des conflits autour de la bande des «Apaches ", dont l'auteur dépeint les activités à travers des portraits de membres. Ces premières études ethnographiques ont souvent été rédigées sur un ton journalistique, à l'image de l'étude des gangs de Boston, conduite par Puffer ${ }^{13}$. Les travaux de Riis sur New York ont, eux, montré les difficultés rencontrées par les nouveaux immigrés - en particulier les Irlandais et les Italiens - à s'intégrer dans l'économie légale ${ }^{14}$. Le travail d'Asbury dans les années 1920 dans la zone de Five Points à New York a établi une distinction entre les membres passagers de gangs et les délinquants plus persistants, qui prenaient part à des activités criminelles plus organisées ${ }^{15}$. Asbury a montré le rôle de la succession ethnique dans ces processus.

L'ouvrage classique de Thrasher, The Gang, publié en 1927 participe de l'école de sociologie de Chicago. Thrasher met l'accent sur le rôle de la culture et des quartiers dans la formation des gangs qui, selon lui, se développent principalement dans des zones «interstitielles ", c'est-à-dire généralement dans des quartiers dont l'état se dégrade et dont la composition ethnique change, favorisant des phénomènes de désorganisation des bidonvilles ethniques. L'une des thématiques centrales de son travail est la diversité des gangs. Il écrit ainsi que "pas un gang ne se ressemble 16 ». Pour lui, de diffus et éphémères, les groupes criminels ont atteint des états fixes et conventionnels dans lesquels les membres jouent des rôles conventionnels.

12. Lewis A., The Apaches of New York, Londres, Dillingham, 1912.

13. Puffer J., op cit., 1912.

14. Riis J., The Battle with the Slum, New York, MacMillan, 1902.

15. Asbury H., Gangs of New York: An Informal History of the Underworld, New York, Paragon House, 1927.

16. Thrasher F. M., " No two gangs are alike ", The Gang, Chicago, Chicago University Press, 1927, p. 5. 
L'étude des jeunes hommes italiens à Boston par William Foote Whyte, devenue une référence en la matière, a également mis l'accent sur la culture du conflit, conséquence de l'exclusion de ces jeunes immigrés européens de la société, en particulier des écoles, des structures familiales et de l'emploi ${ }^{17}$. L'étude des gangs et de la jeunesse délinquante à Boston par Walter Miller met l'accent sur l'isolement culturel des immigrants et de certains groupes ethniques par rapport aux valeurs culturelles majoritaires ${ }^{18}$. Une telle perspective lui permet de conclure que la délinquance est un produit naturel, conséquence de la socialisation et de l'engagement pour des valeurs qui entrent en conflit avec la société conventionnelle. Miller a identifié six problématiques centrales au quotidien dans les quartiers pauvres : le sort, l'autonomie, l'intelligence, la robustesse/dureté, l'excitation et les problèmes.

Les travaux de Short et Strodtbeck à Chicago ont marqué la fin de la période classique ${ }^{19}$. Ces deux chercheurs ont mis l'accent sur la nature groupée des gangs en tant qu'unité d'analyse et ils ont promu l'étude des processus collectifs. Leur recherche a cela de nouveau par ailleurs qu'elle a mis l'accent sur le niveau individuel et sur les changements macro-structurels.

\section{La période interstitielle de l'ethnographie des gangs}

Si les groupes de Los Angeles ont toujours suscité l'intérêt des chercheurs, ceux des villes de la Rust Belt ont également été scrutés au cours de cette période. L'ethnographie des gangs est alors marquée par un fort attrait pour les questions de marginalisation, de genre, de violence, d'entrepreneuriat illicite, et de structure et d'organisation de groupe. Moore, Hagedorn et Vigil analysent tous trois le rôle de la marginalisation économique dans la formation et la structuration des gangs ${ }^{20}$. Le passage d'une économie industrielle à une économie de services a entrainé un fort déclin de l'emploi chez les personnes peu qualifiées, une évolution qui a eu des effets tragiques pour de nombreux individus impliqués dans les gangs. Comprise comme l'exclusion de certains groupes des institutions sociales traditionnelles, telles que l'école ou le marché du travail, une analyse de la désocialisation permet de mieux comprendre ces groupes criminels. Pour Vigil, ces individus ont souffert d'une "double marginalisation ", n'étant par ailleurs pas reconnus dans leurs propres communautés. Cette double mise à l'écart montre le rôle de l'exclusion ethnique et de l'immigration, deux concepts particulièrement pertinents pour les recherches menées en Europe ainsi que pour celles sur les gangs en Amérique centrale et du sud, comme nous le verrons plus tard.

17. Whyte W., Street Corner Society: The Social Structure of an Italian Slum, Chicago, University of Chicago Press, 1943.

18. Miller W., op. cit., 1958.

19. Short J., Strodtbeck F., op. cit., 1965.

20. Moore J., op. cit., 1978 ; Moore J., op. cit., 1991 ; Hagedorn J., op. cit., 1988 ; Vigil J., Barrio Gangs: Street Life and Identity in Southern California, Austin, University of Texas Press, 1988. 
C'est au cours de cette période que la recherche s'est intéressée aux groupes féminins et aux membres en tant qu'individus ${ }^{21}$. On peut citer ici les travaux de Moore, Campbell et Miller sur la question du genre dans les gangs ainsi que, plus généralement, sur les clans féminins ${ }^{22}$. Campbell en particulier, identifie deux rôles occupés par les femmes : celui d'objet sexuel et celui de « témoin » ou de porte-armes ou porte-drogues. Miller s'est attaché à étudier de manière ethnographique et comparative la vie des filles des gangs de Columbus dans l'Ohio et de Saint Louis, dans le Missouri. Il a montré l'influence de l'environnement structurel et de la division genrée des rôles dans leur parcours.

La recherche menée au cours de cette période - en particulier à la fin des années 1980 et au début des années 1990 - s’est par ailleurs beaucoup intéressée à la violence, au trafic de drogue et à la structure des gangs. Comment ces malfaiteurs organisaient-ils la violence et le trafic ? Parmi les bandes étudiées dans diverses villes, on peut citer des groupes afro-américains faiblement organisés à Saint-Louis, les Fremont Hustlers (à majorité afro-américaine) à Kansas City, différents groupes afro-américains dans le Milwaukee postindustriel, des gangs de Portoricains de deuxième génération à Chicago et les Black Kings dans un grand ensemble immobilier de Chicago qui a depuis été désaffecté ${ }^{23}$. Le degré d'organisation a beaucoup varié en fonction des lieux, mais la violence exercée par ces gangs a atteint des niveaux sans précédent et elle a notamment inclus de nombreux éléments de représailles ${ }^{24}$.

La quantité de travaux ethnographiques sur les gangs aux États-Unis a diminué à la fin des années 1990 et on a alors vu émerger des recherches menées au Royaume-Uni et en Europe continentale. La recherche qui avait été conduite au cours des deux périodes que nous venons de décrire a offert les fondements d'une analyse quantitative des groupes criminels et de leurs membres aux États-Unis. Une exception notable à cette tendance reste l'excellent travail mené par Lauger sur le rôle joué par la culture, l’immigration et l'identité de groupe dans les gangs d'Indianapolis ${ }^{25}$.

21. Campbell A., The Girls in the Gang, Cambridge, Blackwell, 1984 ; Moore J., op. cit., 1991 ; Joe K., Chesney-Lind M., «Just Every Mother's Angel: An Analysis of Ethnic and Gender Variations in Youth Gang Membership », Gender and Society, 9, 1995, pp. 409-431 ; Joe K., Hunt G., "Accomplishing Femininity among the Girls in the Gang ", British Journal of Criminology, 41, 2001, pp. 656-78 ; Miller J., One of the Guys: Girls, Gangs, and Gender, New York, Oxford University Press, 2001.

22. Moore J., op. cit., 1991 ; Campbell A., op. cit., 1984 ; Miller W., op. cit., 2001.

23. Decker S., Van Winkle B., op. cit., 1996 ; Fleisher M., Dead End Kids: Gang Girls and the Boys They Know, Madison, Wisconsin University Press, 1998 ; Hagedorn J, op. cit., 1998 ; Padilla F., The Gang as an American Enterprise, New Brunswick, Rutgers, 1992 ; Venkatesh S., Gang Leader for a Day, New York, Penguin Books, 2008.

24. Decker S., "Collective and Normative Features of Gang Violence ", Justice Quarterly, 13, 1996, pp. 243-264 ; Decker S., Pyrooz D., op. cit., 2010.

25. Lauger T., Real gangstas: Legitimacy, reputation, and violence in the intergang environment, New Brunswick, Rutgers University Press, 2012. 


\section{L'ethnographie des gangs à l'époque contemporaine}

Le nombre et la qualité des études ethnographiques menées en Europe continentale et au Royaume-Uni ont augmenté de manière considérable au cours de la période dite contemporaine. Il est important de noter que si de telles études se sont également développées en Amérique Centrale et du Sud, nous les traiterons comme un phénomène unitaire dans une section dédiée. Le travail de James Patrick, A Glasgow Gang Observed publié en 1973, est devenu un classique comme nous l'avons déjà mentionné, car il a permis de montrer l'existence de gangs violents au Royaume-Uni à un moment où peu de travaux étaient entrepris en dehors des États-Unis. Les deux forces principales des recherches menées au cours de la période contemporaine s'incarnent dans l'intérêt qu'elles portent aux contextes structurels, mais aussi culturels. En effet, s'intéresser aux aspects structurels des mouvements de populations a permis de comprendre la marginalisation et l'exclusion subies, ainsi que les conflits ethniques et la transmission culturelle.

L'histoire, les modèles migratoires, les politiques et les effets de ces mouvements de population en Europe sont des sujets de recherche constants ${ }^{26}$. La migration européenne reflète l'impérialisme des nations de l'Europe occidentale, en particulier du point de vue des colonies établies en Afrique, en Asie et en Amérique du Sud. La relation particulière qui peut exister entre les colonisateurs et les populations autochtones est une question essentielle, dès lors que les individus ont été en mesure de voyager entre l'État européen et l'ancienne colonie. Les relations sont souvent restées étroites entre les pays, à l'image du Royaume-Uni avec l'Inde, le Pakistan et les Caraïbes, la France avec l'Algérie, le Maroc et le Sénégal, le Portugal avec le Brésil et l'Angola, et les Pays-Bas avec l'Indonésie et le Surinam. Les États européens ont d'ailleurs souvent mis en place des voies légales d'immigration pour les résidents des anciennes colonies.

Trois facteurs ont façonné les modèles migratoires européens : d'abord, la croissance économique rapide du vieux continent à cette époque a créé un besoin de main-d'œuvre que ces États sans croissance démographique n'étaient pas en mesure de combler seuls. Dans certains cas, les pays européens ont recruté des "travailleurs invités ", notamment pour les emplois à faible salaire ${ }^{27}$. Si cette relation devait être temporaire, dans de nombreux cas, pourtant, elle s'est pérennisée. Ensuite, la chute du mur de Berlin et l'effondrement de la République socialiste soviétique et partant du contrôle des anciens États

26. Voir Hammar T., European Immigration Policy: A Comparative Study, Cambridge, Cambridge University Press, 1985 ; Jacobson D., The Immigration Reader: American in a Multidisciplinary Perspective, New York, Wiley, 1998.

27. Obdeijn H., de Mas P., Hermans P., De Geschiedenis van Marokko, Amsterdam, Bulaaq, 2002. 
satellites ont entrainé des mouvements de population entre ces pays et l'Europe occidentale ${ }^{28}$. Enfin, du fait d'un contexte instable, voire dangereux, dans les anciennes colonies, des populations africaines, moyen-orientales et asiatiques ont également cherché l'asile et du travail en Europe ${ }^{29}$.

Les États européens comptent désormais un certain nombre de groupes immigrés de première et de deuxième générations. Généralement jeunes, ces populations non blanches, de culture non européenne, sont concentrées dans des zones urbaines marginalisées.

\section{Des conflits ethniques et culturels}

L'un des principaux résultats de la recherche ethnographique classique et contemporaine sur les gangs est le rôle central que jouent les peurs et les conflits liés à l'arrivée de nouveaux groupes d'immigrés. Les cultures sont à la fois réactives et capables de s'adapter, ce qui fait que de nombreux jeunes se sont trouvés face à des impératifs culturels concurrents et qu'ils ont eu à choisir entre une loyauté envers la culture de leurs parents ou envers celle de leurs pairs. Pour de nombreux jeunes, le gang s'est avéré une option attirante qui leur a permis d'échapper à la culture de leurs parents, pas tout à fait « compatible » avec leur nouvel environnement.

Le déclin de la recherche ethnographique sur les gangs aux États-Unis est allé de pair avec une amélioration de la qualité et du nombre de ce type de travaux dans le contexte européen. Van Gemert a notamment mené une recherche de terrain importante auprès de jeunes immigrés marocains et turcs à Amsterdam, dans laquelle il observe des expériences d'arrestation hétéroclites ${ }^{30}$. Les jeunes hommes marocains sont par exemple surreprésentés dans les statistiques de la police et sont plus souvent membres de gangs que ne le sont les jeunes Turcs. En dépit de ressemblances de statut (immigré) et d'une marginalisation subie, Van Gemert montre que les jeunes de ces deux origines prennent des chemins différents du point de vue de leur participation à des bandes ou à des activités de délinquance. Il identifie des différences culturelles entre les deux groupes communautaires, telles que des modèles de croyance et de pratiques, en particulier chez les jeunes.

28. Andreas P., Snyder T., The Wall around the West: State Borders and Immigration Controls in North America and Europe, Lanham, Rowman \& Littlefield, 2000.

29. Van Wijk J., Luanda-Hollanda: Irreguliere (Asiel) Migratie van Angola Vaa, Nederland, Nijmegen, Wolff Legal, 2006 ; Engbersen G., Van der Leun J., Boom J., " The Fragmentation of Migration and Crime ", in Tonry M., Bijleveld C. (eds.), Crime and Justice in the Netherlands, vol. 35, Crime and Justice, A Review of Research, Chicago, University of Chicago Press, 2007.

30. Van Gemert F., Fleisher M., «In the Grip of the Group: Ethnography of a Moroccan Street Gang in the Netherlands ", in Decker S., Weerman F. (eds.), European Street Gangs and Troublesome Youth Groups, Lanham, Alta Mira, 2005. 
L'ouvrage collectif dirigé par Weitekamp s'attache à analyser le rôle de la culture chez les Russes rapatriés d'origine allemande ${ }^{31}$. Ces individus connus sous le qualificatif d'Aussiedler avaient migré vers la Russie à la fin de la Seconde Guerre mondiale, lorsque l'Allemagne avait été scindée en deux. La plupart des anciens citoyens allemands avaient ainsi perdu leurs coutumes et leur langue et les avaient remplacées par la culture et la langue de leur pays d'accueil. À leur retour en Allemagne, ces Aussiedler se sont trouvés en conflit avec les Allemands et avec d'autres groupes ethniques vivant sur place. Leurs expériences correspondent au modèle de la double marginalisation décrite par Vigil 32 .

Les notions de culture et d'ethnicité sont au cœur de l'analyse dans de nombreux travaux européens sur les gangs. Le gang, en tant que groupe, semble secondaire dans l'analyse. Un exemple très instructif est le travail ethnographique mené par Bucerius sur des hommes musulmans vivant à Francfort, en Allemagne ${ }^{33}$. Plus d'une cinquantaine d'hommes de la deuxième ou la troisième génération immigrée d'obédience musulmane ont été observés et interrogés. Si plus de la moitié de ces individus étaient d'origine turque, tous étaient nés en Allemagne et prenaient part au trafic de drogue dans la rue. Leur éducation et leur culture musulmane et turque ont créé une combinaison culturelle unique des concepts «d'honneur » et de "pureté », qui forment la base de ce qui fait un «bon croyant» du point de vue des comportements et des convictions religieuses. Le processus d'adaptation à ce statut de marginal (ils n'ont aucun droit civique en Allemagne) a contraint leurs valeurs culturelles à une série d'adaptations, en particulier en ce qui concerne la vente de drogue. Ils se justifiaient d'ailleurs en précisant qu'ils vendaient les «drogues les moins nocives » et uniquement aux «clients appropriés». Selon Bucerius, les hommes de son échantillon rééquilibrent leur comportement entrepreneurial par des valeurs culturelles dont les racines sont à trouver dans leur religion, même s'ils adaptent ces croyances. Ces arrangements ont créé une "culture hybride » qui a permis de générer un équilibre entre les croyances et la manière dont ils étaient traités en Allemagne ${ }^{34}$. Bucerius a caractérisé l'évolution et l'adaptation des valeurs religieuses et culturelles comme une conséquence de l'aliénation des groupes ethniques de leurs cultures d'origine et d'accueil.

31. Weitekamp E., Hans-Jurgen K., Kerstin R., «Why Do Young Male Russians of German Descent Tend to Join or Form Violent Gangs? ", in Decker S., Weerman F. (eds.), European Street Gangs and Troublesome Youth Groups, Lanham, Alta Mira, 2005.

32. Vigil J., A Rainbow of Gangs: Street Life and Identity in the Mega-City, University of Texas Press, 2002.

33. Bucerius S., « What Else Should I Do? Cultural Influences on the Drug Trade on Migrants in Germany ", Journal of Drug Issues, 37, 2007, pp. 673-697.

34. Ibid., p. 682. 
L'étude d'Aldridge et Medina est l'une des plus importantes d'Angleterre. Menée pendant près de six ans dans une grande ville, cette recherche a permis d'identifier la complexité des ethnographies de populations marginalisées dans des environnements marqués par des niveaux de violence élevés ${ }^{35}$. L'origine ethnique des premiers groupes examinés était mixte et la composition principalement masculine. Le trafic de drogue était « l'activité dominante, celle qui définissait le gang, ou presque 36 ». L'adhésion au gang était de courte durée et ne menait que rarement à la criminalité chez l'adulte ou à des activités criminelles plus formelles ou organisées. Si le statut d'immigré n'est pas une caractéristique centrale chez les individus décrits, les deux chercheurs notent toutefois que la présence des bandes de malfaiteurs a eu des conséquences culturelles pour les jeunes des quartiers. Victimes d'une surveillance accrue et de la menace d'une victimisation, ces individus ont ainsi pu être poussés à l'exclusion des institutions sociales légitimes.

John Pitts a conduit une étude des gangs de Waltham Forest en ayant recours à une méthode mixte. Dans cette zone du nord-ouest de Londres, dont la population est principalement pauvre et noire ou issue de minorités ethniques ${ }^{37}$, les niveaux de trafic de drogue et de violence sont élevés. Pitts révèle l'existence d'un paradoxe à propos des groupes européens et britanniques : en effet, si des symboles généralement rattachés aux activités des gangs sont visibles (graffitis, trafic de drogue, affiliation des jeunes), en réalité, peu de chercheurs ont qualifié ces groupes de « gangs », leur préférant d'autres termes ou expressions, comme celle de « collectivités urbaines » (que l'on retrouve notamment dans les conclusions de Hallsworth et Young) ${ }^{38}$. Pour John Pitts toutefois, les gangs de Waltham Forest ont de nombreux points communs avec ceux des États-Unis et ils doivent donc porter cette dénomination. Dans ce quartier londonien, comme ailleurs, la race et l'origine ethnique contribuent à la pauvreté et à l'isolement social, une conclusion confirmée par les travaux de John Pitts. Celui-ci montre justement que c'est l'intersection entre ethnicité et culture de gang qui importe et que la mondialisation du style des gangs de jeunes Américains n'est qu'un élément parmi d'autres du développement et de la diffusion des gangs à Londres. En effet, la culture "yardie ", originaire de Kingston, en Jamaïque, a également joué un rôle très important dans le processus. Pitts identifie des «territorialités agressives» au sein des groupes - ce qui les différencie d'autres gangs britanniques ou

35. Aldridge J., Medina J., Ralphs R., « Dangers and Problems of Doing 'Gang' Research in the UK », in Van Gemert F., Peterson D., Lien I.-L. (eds.), Street Gangs, Migration and Ethnicity, Londres, Willan, 2008.

36. Ibid., p. 6.

37. Pitts J., «Reluctant Gangsters: Youth Gangs in Waltham Forest », Report to the University of Bedfordshire, Bedford, UK, 2007.

38. Hallsworth S., Young T., "Getting Real about Gangs », Criminal Justice Matters, 55, 2004, pp. 12-13. 
d'Europe continentale - ainsi qu'un certain nombre de " gangsters réticents » parmi les jeunes qu'il a observés.

Le travail ethnographique de James Densley décrit à la fois la structure organisationnelle des gangs londoniens et leurs activités ${ }^{39}$. Il a notamment montré le rôle de la culture de groupe et celui de la génération de profits. Cette recherche est l'une des plus remarquables du point de vue de l'ampleur du travail de terrain mené et des nombreux aspects de la vie des gangs que Densley a été en mesure d'étudier.

\section{La transmission culturelle}

Comment se fait-il qu'un gang américain comme celui des Crips existe également aux Pays-Bas ou en Norvège ? C'est par la culture que Van Gemert et Lien ont expliqué cette présence de gangs aux symboles et aux croyances nord-américains dans les sociétés néerlandaise et norvégienne ${ }^{40}$. Pour eux, la transmission culturelle de symboles s'est faite par les médias, et même si les symboles et les noms sont identiques, les Crips des États-Unis diffèrent de leurs homologues néerlandais, qui sont mal organisés, participent assez peu au trafic de drogue et ne réclament pas de territoire. Finalement, les deux groupes partagent plus un style qu'un mode d'organisation ou des activités, ce que l'on observe par ailleurs dans la transmission de la culture de gang aux ÉtatsUnis ${ }^{41}$.

De nombreux chercheurs ont relié la mondialisation des gangs à des facteurs culturels tels que des reportages diffusés dans les médias, des vidéos, et la participation à des réseaux sociaux. Decker, Van Gemert et Pyrooz ont montré que des processus similaires existent, par lesquels des pratiques culturelles américaines, qui incluent la vie dans un gang, sont apparues comme une source de comportement culturellement transmise et imitée par les jeunes dans de nombreux pays ${ }^{42}$. La mondialisation transmet une grande variété de messages, et la culture des jeunes est sensible à l'adoption de formes de remises en question des normes majoritaires.

39. Densley, J. C., How Gangs Work: An Ethnography of Youth Violence, Londres, Palgrave Macmillan, 2013.

40. Van Gemert F., "Crips in Orange; Gangs and Groups in the Netherlands ", in Klein W., Kerner H., Maxson C., Weitekamp E. (eds.), The Eurogang Paradox: Street Gangs and Youth Groups in the U.S. and Europe, Dordrecht, Kluwer, 2001 ; Lien I., "The Concept, Honor, Conflict, and Violent Behavior among Youths in Oslo ", in Klein W., Kerner H., Maxson C., Weitekamp E. (eds.), The Eurogang Paradox: Street Gangs and Youth Groups in the U.S. and Europe, Dordrecht, Kluwer, 2001; Lien I., « Nemesis and the Achilles Heel of Pakistani Gangs in Norway ", in Van Gemert F., Peterson D., Lien I. (eds.), Street Gangs, Migration and Ethnicity, Londres, Willan, 2008.

41. Maxson C., Gang Members on the Move, Washington, US Department of Justice, Office of Juvenile Justice and Delinquency Prevention, 1998.

42. Decker S., Van Gemert F., Pyrooz D., "Gangs, Migration and Crime: The Changing Landscape in Europe and the USA », Journal of International Migration and Integration, 10, 2009, pp. 393-408. 
Brotherton s'est intéressé à cette diffusion transnationale du comportement et de la culture des gangs ${ }^{43}$. Il a notamment observé les liens entre les Latin Kings aux États-Unis et les membres du gang à l'étranger, comme on peut par ailleurs le lire dans l'ouvrage collectif dirigé par Feixa, qui avait identifié un lien dans la diffusion de la culture de gang aux États-Unis, en Espagne et en Amérique du Sud 44. Peterson, Lien et Van Gemert considèrent que les traditions culturelles jouent un rôle particulièrement important quand de nouveaux groupes d'immigrés se trouvent marginalisés, comme c'est le cas des Pakistanais en Norvège, des communautés musulmanes en Suède et des jeunes latino-américains en Espagne ${ }^{45}$. Des gangs comme la Mara Salvatrucha (également appelée MS-13) et Barrio 18, ont attiré l'attention de la police et des médias au niveau international ${ }^{46}$. Ces gangs fondés à Los Angeles sont présents dans un grand nombre d'États nord-américains, mais aussi à l'étranger ${ }^{47}$. Des immigrés d'Amérique centrale auraient ainsi créé de nouveaux groupes MS-13 à divers endroits des États-Unis 48. En outre, l'exclusion massive de nombreux membres de groupes de Los Angeles en Amérique Centrale aurait joué un rôle certain dans la globalisation des gangs ${ }^{49}$, comme nous le verrons plus en détail dans la section intitulée « La révolution Rodgers».

Selon d'autres lectures, critiques de cette perspective, ce sont les conditions économiques désastreuses et une violence répandue au Salvador qui ont créé une situation dans laquelle les gangs nord-américains ont pu être perçus comme des modèles attirants pour les membres de gangs locaux préexistants, comme les pandillas ${ }^{50}$. Il est évident que les images culturelles des gangs nordaméricains ont été importées et adaptées à la culture locale. En revanche, il est plus difficile de savoir si l'émergence de gangs transnationaux est véritablement le résultat d'un mouvement coordonné des membres de ces gangs. Pour McGuire, il peut s'agir de "mouvements des styles et des pratiques des gangs plus que de leurs membres 51 ». Les expulsions ont certes pu jouer un rôle, mais la diffusion de la culture (les pratiques, les symboles et les idées) n'est pas à négliger dans cette dissémination, loin de là.

43. Brotherton D., «Proceedings from the Transnational Street Gang/Organization Seminar ", Crime, Media and Culture, 3, 2007, pp. 372-381.

44. Feixa C., Canells N., Porzio L., Recio C., Biliberti L., "Latin Kings in Barcelona », in Van Gemert F., Peterson D., Lien I.-L. (eds.), Street Gangs, Migration and Ethnicity, Londres, Willan, 2008.

45. Peterson D., Lien I., Van Gemert F., Street Gangs, Migration, and Ethnicity, Devon, Willan, 2008.

46. Franco C., « The MS-13 and 18th Street Gangs: Emerging Transnational Gang Threats? », http://assets.opencrs.com/rpts/RL34233_20080130.pdf, Accessed July 14, 2008.

47. Savenije W., Maras y Barras. Pandillas y Violencia en los Barrios Marginales de Centroamérica, San Salvador, FLACSO, 2009.

48. Johnson S., Muhlhausen D., " North American Transnational Youth Gangs: Breaking the Chain of Violence. ", The Heritage Foundation : http://www. heritage.org/Research/UrbanIssues/bg1834.cfm, 2005 ; Papachristos A., " Gang World», Foreign Policy, 147, 2005, pp. 49-55.

49. Rodgers D., Baird A., "Understanding Gangs in Contemporary Latin America » in Decker S. and Pyrooz D. (eds.), The Handbook of Gangs, New York, Wiley, 2015. 
À mesure que les gangs se sont diffusés dans de nouvelles parties du monde, la migration, les conflits culturels, la marginalisation et la transmission culturelle ont tous été identifiés dans les travaux contemporains d'ethnographie comme ayant influé sur les phénomènes liés aux gangs. Il existe de nombreux éléments dans les ethnographies récentes sur les bandes de malfaiteurs européens, qui prouvent qu'il existe une variabilité considérable dans la structure des gangs du continent ${ }^{52}$. Ces travaux se sont intéressés à divers groupes à Amsterdam et La Haye (Pays-Bas), à Francfort (Allemagne), à Gênes (Italie), à Manchester (Grande Bretagne), à Oslo (Norvège), en Slovénie, mais aussi en Russie et au Maroc. Tous ont révélé la grande hétérogénéité des groupes sur le continent européen, qui finalement, et de façon assez ironique, rappelle la situation nord-américaine, où les gangs et leurs membres varient considérablement du point de vue de la violence exercée, de la structure du groupe, de son organisation, de la nature de la délinquance, du genre, de la race, de l'ethnicité, de la culture et du statut.

50. Savenije W., op. cit., 2009.

51. McGuire C., "Working Paper on Central American Youth Gangs in the Washington, D.C., Area ", Washington Office Ln America (WOLA), http://www.wola.org/index.php?option=com_content\&task=viewp\&id=272\&Ite mid=2, 2007, p. 27.

52. Dekleva B., "Gang-Like Groups in Slovenia ", in Klein M., Kerner H., Maxson C., Weitekamp E. (eds.), The Eurogang Paradox: Street Gangs and Youth Groups in the U.S. and Europe, Dordrecht, Kluwer, 2001 ; Duran, R. J., Gang Life in Two Cities: An Insider's Journal, New York, Columbia University Press, 2013 ; Fraser, A., Urban Legends: Gang Identity in the Post-Industrial City, Oxford, Oxford University Press, 2015 ; Gruter P., Versteegh P., "Towards a Problem-Oriented Approach to Youth Groups in The Hague », in Klein M., Kerner M., Maxson C., Weitekamp E. (eds.), The Eurogang Paradox: Street Gangs and Youth Groups in the U.S. and Europe, Dordrecht, Kluwer, 2001 ; Lien I., op cit., 2001; Lien I., "Criminal Gangs and Their Connections: Metaphors, Definitions and Structures ", in Decker S.,Weerman F. (eds.), European Street Gangs and Troublesome Youth Groups, Lanham, Alta Mira, 2005 ; Lien I., op cit., 2008 ; Mares D., « Gangstas or Lager Louts? Working Class Street Gangs in Manchester », in Klein M., Kerner H., Maxson C., Weitekamp E. (eds.), The Eurogang Paradox: Street Gangs and Youth Groups in the U.S. and Europe, Dordrecht, Kluwer, 2001 ; Salagev A., « Evolution of Delinquent Gangs in Russia, » in Klein M., Kerner H., Maxson C., Weitekamp E. (eds.), The Eurogang Paradox: Street Gangs and Youth Groups in the U.S. and Europe, Dordrecht, Kluwer. 2001 ; Tertilt H., "Patterns of Ethnic Violence in a Frankfurt Street Gang », in Klein M., Kerner H., Maxson C., Weitekamp E. (eds.), The Eurogang Paradox: Street Gangs and Youth Groups in the U.S. and Europe, Dordrecht, Kluwer, 2001 ; Gatti U., Angelini F., Marengo G., Melchiorre N., Sasso M., « An Old-Fashioned Youth Gang in Genoa », in Decker S. and Weerman F (eds.), European Street Gangs and Troublesome Youth Groups, Lanham, Alta Mira, 2005 ; Van Gemert F., Fleisher D., op cit., 2005 ; Weitekamp E.G., Reich K., Kerner H.J., «Why do young male Russians of German descent (Aussiedlers) tend to join or form gangs where violence plays a major role ", in S. H. Decker and F. M Weerman (eds.), European street gangs and troublesome youth groups: Findings from the Eurogang research program, Oxford, AltaMira Press, 2005, pp. 103-136 ; Pitts J., op cit., 2007 ; Aldridge J., Medina J., Youth Gangs in an English City: Social Exclusion, Drugs and Violence, University of Manchester, In Research Report, 2008, ESRC RES-000-23-0615. 


\section{La révolution Rodgers}

Nous qualifions cette tendance de la recherche ethnographique sur les gangs de "révolution Rodgers ", car elle doit beaucoup à la méthodologie et à l'approche théorique et politique de Dennis Rodgers ${ }^{53}$. Ce dernier s'est servi de méthodes ethnographiques dans l'étude des gangs d'Amérique centrale dès $19977^{54}$. Au-delà de ses recherches (aussi considérables et importantes qu'elles puissent être), c'est un ensemble de travaux de qualité sur l'Amérique latine (y compris l'Amérique Centrale et du Sud, ainsi que les Caraïbes) qu'il a inspirés et qui ont été menés depuis la fin du siècle dernier. Le qualificatif de "révolution Rodgers » s'applique à cet ensemble. C'est en partageant ses méthodes, son temps et ses connaissances avec ses pairs, y compris avec des chercheurs plus jeunes résidant dans cette partie du monde, que $\mathrm{D}$. W. Rodgers a marqué les travaux ethnographiques de cette période. D’une certaine manière, cette influence est aussi marquante et importante que les travaux en eux-mêmes. Cette génération d'ethnographes, fortement marquée par l'importance de la recherche de terrain, est composée d'anciens étudiants de Rodgers, inspirés par ses travaux, mais aussi de chercheurs ayant collaboré avec lui. Le travail, difficile et parfois dangereux, exige de savoir naviguer entre les langues, les cultures et des environnements internationaux différents. Si nombre de ces chercheurs ont leurs attaches en Europe, les « locaux » sont également nombreux, et on peut ainsi affirmer que Rodgers a d'une certaine manière suscité l'émergence une génération de chercheurs indigènes.

Au moins cinq principes généraux sur les gangs peuvent être déduits de ces travaux. D'abord, les gangs de la région sont situés et ils reflètent la culture, la structure et les histoires locales. Ensuite, les gangs sont variables et dynamiques, c'est-à-dire qu'ils ne forment pas un bloc et qu'ils peuvent se transformer dans le temps. Troisièmement, les gangs d'Amérique latine sont complexes et il est donc difficile de les caractériser à partir des structures, des activités ou des formulations culturelles d'autres régions. Quatrièmement, la violence est un élément central des gangs, notamment parce qu'ils sont impliqués dans le commerce de narcotiques (vente au détail, en gros, ou les deux). Enfin, ces bandes de malfaiteurs sont de plus en plus impliquées dans des actes de grande criminalité, y compris par l'usage d'armes de plus en plus sophistiquées.

Il n'est pas aisé d'identifier la première étude de terrain des gangs dans la région, même si Rodgers cite le travail de Deborah Levenson (1988) sur les gangs de rue au Guatemala comme étant une étude «fondatrice 55 ». Il établit

53. L'auteur de cet article entretient avec Dennis Rodgers des liens purement professionnels : outre la publication d'un article de Rodgers dans un ouvrage sous sa direction, il a rencontré Rodgers dans plusieurs congrès.

54. Rodgers D., "Living in the Shadow of Death: Gangs, Violence and Social Order in Urban Nicaragua, 1996-2002 ", Journal of Latin American Studies, 38, 2006, pp. 267-292. 
un lien entre ce travail et celui de Núñez sur le Guatemala, le Salvador et le Nicaragua ${ }^{56}$. D’autres études ont suivi, sur le Salvador et le Honduras. Ces premières incursions sur le terrain ont donné lieu à d'autres travaux menés par plus de quarante chercheurs dans au moins neuf pays (Brésil, Colombie, Équateur, Salvador, Guatemala, Honduras, Nicaragua, Mexique et Venezuela) et vingt villes différentes. Comme nous l'avons déjà mentionné, ce sont principalement des universitaires européens qui ont mené les premières études. Reste que la logistique nécessaire à l'installation d'une base en Amérique Latine, à la maitrise de la langue et des codes est considérable, même si certains chercheurs, comme Hume, continuent de mener des projets de recherche en Amérique Centrale ${ }^{57}$. La dépendance au travail de terrain jusque récemment dans cette région du monde est largement liée au fait que l'accès aux institutions (écoles, prisons, centres pour la jeunesse) est difficile et que les statistiques officielles sont sommaires et peu fiables 58 . C'est donc presque uniquement en allant sur le terrain qu'on peut recueillir des données sur le sujet étudié.

Nombre des grands thèmes abordés dans la recherche sur les gangs reflètent l'attention portée par Rodgers à la notion de culture. Il écrit : «(ils) sont reliés à la pauvreté de manière contextuelle, mais pas nécessairement fortuite ; des liens structurels peuvent être établis entre l'émergence de gangs après la guerre froide et la longue histoire de l'insurrection et de la résistance à l'oppression dans cette région du monde et il n'existe pas de facteur unique qui permettrait d'expliquer la participation à un gang ou la vie des individus après qu'ils ont quitté le gang 59 ». Comme Rodgers et Baird 60 le soulignent, les gangs sont devenus le principal épouvantail dans la région, remplaçant ainsi le communisme, et sont accusés d'une variété de maux économiques et poli-

55. Levenson D., Figueroa N. M., Maldonado Castilla, M. Y., Por símismos: Un estudio preliminar de las "maras" en la ciudad de Guatemala, Cuaderno de Investigación, n 4, Guatemala, Asociación para el Avance de las Ciencias Sociales en Guatemala (AVANCSO), 1998.

56. Núñez J. C., De la Ciudad al Barrio: Redes y Tejidos Urbanos en Guatemala, El Salvador y Nicaragua, Ciudad de Guatemala, Universidad Rafael Landívar/ PROFASR, 1996.

57. Hume M., «It's as if you don't know, because you don't do anything about it': Gender and violence in El Salvador ", Environment and Urbanization, 16, 2, 2004, pp. 63-72.

58. Maguire, Harriott A., Katz C., Gangs in the Caribbean: Responses of State and Society, Jamaica, University of West Indies Press, 2015.

59. Notre traduction. Baird A., « Negotiating Pathways to Manhood: Rejecting Gangs and Violence in Medellín's Periphery ", Journal of Conflictology, 3,1, 2012a, pp. 30-41 ; Baird A., "The violent gang and the construction of masculinity amongst socially excluded young men ", Safer Communities: A Journal of Practice, Opinion, Policy and Research, 11, 4, 2012, pp. 179-190 ; Baird A., "¿Héroes Olvidados? Activismo de la sociedad civil y las políticas de juventud en Medellín », in Baird A., Serrano F. (eds.), Paz Paso a Paso: Una mirada desde los Estudios de Paz a los Conflictos Colombianos, Bogotá, Pontificia Universidad Javeriana, 2013 ; Baird A., « Duros and Gangland Girlfriends: Male Identity and Gang Socialisation in Medellín », in Auyero J., Bourgois P., Schepper- Hughes N. (eds.), Violence at the Urban Margins, Oxford, Oxford University Press, 2015 ; Rodgers D., Baird A. , « Understanding Gangs in Contemporary Latin America », in Decker S. and Pyrooz D. (eds.), The Handbook of Gangs, New York, Wiley, 2015.

60. Rodgers D., Baird A., op. cit., 2015. 
tiques ${ }^{61}$. On ne trouve en effet guère de discussion sur les bandes criminelles de la région dans laquelle celles-ci ne soient pas accusées de tel ou tel problème social.

Le premier gang impliqué dans ce processus de diabolisation est la Mara Salvatrucha (MS-13). S'ils sont actifs au Salvador, au Guatemala et au Honduras, ces groupes trouvent leurs origines aux États-Unis, à Los Angeles en particulier. Après de nombreuses années de résidence en Amérique du Nord, suffisantes pour être devenus membres et avoir acquis une forte affiliation au groupe, certains membres ont été renvoyés dans leurs pays d'origine. Ces gangs apparaissent comme étant très structurés, extrêmement violents et dotés de tentacules assez longues pour relier le Triangle du Nord à de nombreuses villes nord-américaines, à tel point qu'il existe un groupe d'intervention spécial (le MS-13 Task Force) au sein du Federal Bureau of Investigation (FBI) pour traquer et combattre les activités de ces gangs. En Amérique Centrale, leurs principaux rivaux sont les pandillas (un terme qui sert à qualifier les groupes criminels nés dans la région). La rivalité est souvent brutale et violente et les deux groupes sont impliqués dans de multiples activités de grande criminalité. Rodgers et Baird soulignent toutefois que les réalités de tels collectifs sont complexes et entrelacées avec des problèmes liés au retour forcé dans leur pays après avoir établi une appartenance à un gang lors du séjour à Los Angeles 62.

De nombreuses recherches de terrain sur les membres de gangs ont été menées au Brésil, pays le plus grand et le plus peuplé d'Amérique latine. Peutêtre cela est-il lié à la longue tradition d'études sur la violence urbaine, en particulier dans les favelas, même si d'aucuns spéculent que Rio de Janeiro attire de nombreuses recherches internationales du fait de ses multiples attraits ${ }^{63}$. Le Brésil aurait une très longue histoire des groupes criminels, notamment avec les maltas dès les années 1800 64. Sa proximité géographique avec la culture et la production de cocaïne fait que le Brésil voit passer de grands volumes de drogue et a même servi de plateforme de transit au cours de certaines périodes. La présence d'armes et d'un produit de valeur (la drogue) rend le contexte favorable à l'éruption de la violence. Si au commencement les gangs étaient rattachés à des quartiers, leur portée et le niveau de la violence ont progressivement augmenté. À Rio de Janeiro comme à Sao Paulo, les prisons ont joué un rôle majeur dans l'expansion des gangs et de leur influence ${ }^{65}$.

61. Ibid.

62. Ibid.

63. Ibid.

64. Les maltas étaient des groupes qui erraient sur les docks dans les années 1800 et avaient un comportement proche de celui des groupes qui sont aujourd'hui qualifiés de gangs. Voir Rodgers D., Baird A., op. cit., 2015.

65. Butler M., Slade G., Dias C. N., «Self-Governing Prisons: Prison Gangs in an International Perspective », Trends in Organized Crime, 2018 ; Denyer Willis G., «Deadly Symbiosis? The 
Les groupes criminels qui existent en Amérique latine partagent un certain nombre de caractéristiques et d'influences. L'instabilité de l'économie et des États a permis la création de vides dans lesquels ont pu s'engouffrer les gangs, leur laissant jouer un rôle important en matière de gouvernance non étatique par l'imposition violente de leur volonté. Rodgers et Baird ont su montrer que les limites qui existent entre les bandes plus organisées et celles reliées à un quartier, généralement moins structurées, sont souvent troubles. C'est-à-dire, selon ces deux chercheurs, qu'une certaine fluidité existe, qui permet à des groupes de fonctionner de manière très organisée dans des situations spécifiques, mais pas dans d'autres. En outre, l'émergence d'une culture des jeunes plus globale qu'auparavant a rendu possible la diffusion vers l'Amérique latine d'une certaine iconographie des gangs et des gangsters, de la même manière que les styles des gangsters américains étaient parvenus jusqu'en Europe quelques décennies plus tôt. Dans chaque cas, le pays d'importation a intégré le «style » en cohérence avec sa propre culture et la culture de ses jeunes. Un travail ethnographique approfondi a été réalisé en Colombie ${ }^{66}$, permettant d'observer que les gangs de ce pays entretenaient des relations avec d'importantes organisations de contrebande, souvent interchangeables. À l'inverse, très peu de recherches ont été menées sur les groupes équatoriens jusqu'à une date récente, avec notamment les travaux de Carrion, Torres et Cerbino. Comme dans d'autres États sud-américains, les gangs se divisent en deux factions principales : les naciones (plutôt organisés, avec une base assez large) et les pandillas (faiblement structurés, locaux) ${ }^{67}$. Torres a notamment étudié les Latin Kings, dont un certain nombre de membres sont des expulsés des États-Unis qui ont réimporté leur style et leur mode d'organisation ${ }^{68}$.

Les gangs de rue existent au Mexique depuis les années 1950 au moins. Les bandas, les gangs mexicains, ont été initiés dans une tentative de combler le vide laissé par des institutions sociales qui ne fonctionnaient plus de manière efficace. Ces groupes sont passés de gangs de quartiers qui offraient aux jeunes un certain sens d'appartenance et des objectifs partagés, à des groupes plus

PCC, the State and the Institutionalization of Violence in São Paulo ", in G. A. Jones, D. Rodgers (eds.), Youth Violence in Latin America: Gangs and Juvenile Justice in Perspective, New York, Palgrave, 2009 ; Dias C., Salla F., « Organized Crime in Brazilian Prisons: The Example of the PCC ", International Journal of Criminology and Sociology, 2, 2013, pp. 397408.

66. Baird A., op cit., 2012a ; Baird A., op cit., 2012b ; Rubio M., De la pandilla a la mara: pobreza, educación, mujeres y violencia juvenil, Bogotá, Universidad Externado de Colombia, 2007; Perea Restrepo C. M., Con el Diablo adentro: pandillas, tiempo paralelo y poder, México, Siglo Veintiuno Editores, 2007.

67. Carrión, F., " De la violencia urbana a la convivencia ciudadana », in Carrión F. (eds.), Seguridad ciudadana: ¿espejismo o realidad?, Quito, Flacso, OMS, OPS, 2002, pp. 13-58; Cerbino M., El Lugar de la Violencia: Perspectivas Críticas sobre Pandillerismo Juvenil, Quito, FLACSO/Santillana/Taurus, 2011 ; Torres, A., « Pandillas y Naciones en Ecuador: diagnóstico de situación », Boletín Cindad Segura, 3, 2006, pp. 4-9.

68. Torres A., op. cit., 2006. 
organisés prenant part à des crimes graves, en particulier au trafic de drogue et à des activités violentes ${ }^{69}$.

Cet état de l'art de l'ethnographie des gangs nous a permis de remettre ces travaux en perspective, du point de vue géographique, mais aussi de leur évolution. Nous avons ainsi présenté quatre périodes de recherche distinctes : la classique, l'interstitielle, la contemporaine et celle que nous qualifions de « révolution Rodgers ». La recherche nord-américaine a dominé les deux premiers cycles, tandis que la recherche européenne a dominé la troisième ère et celle d'Amérique latine la quatrième.

Nous avons ouvert cet article en soulignant que l'étude des gangs s'est beaucoup faite en usant de méthodes ethnographiques et d'enquêtes de terrain : l'ère classique s'est intéressée aux expériences de la première et de la deuxième générations de groupes ethniques d'origine européenne arrivés aux États-Unis à partir du début du XXe siècle. Ces études sont restées très descriptives et se sont intéressées aux caractéristiques de contexte comme l'ethnicité, le statut d'immigré, le genre et la structure familiale. En plus de ces caractéristiques, ces premières enquêtes se sont intéressées aux structures des bandes ainsi qu'à leur implication dans des activités criminelles ou légales. La culture en tant que telle n'était que rarement mentionnée et ces travaux restaient très silencieux sur le rôle des valeurs dans la formation des groupes ou dans leurs activités.

Cette période a été suivie de la période dite «interstitielle », une ère de transition entre l'époque classique et l'époque contemporaine, allant des années 1960 à la fin du XX $x^{\mathrm{e}}$ siècle. Cette période a connu une forte croissance du nombre de travaux ethnographiques se concentrant sur les gangs et leurs membres. Les groupes afro-américains ont suscité le plus grand intérêt, et plus particulièrement leur exclusion des institutions et leur concentration dans des zones urbaines pauvres, ainsi que leurs activités criminelles - en particulier les actes de violence et le trafic de drogue. La plupart des bandes étaient à l'époque faiblement organisées et n'avaient qu'un faible contrôle du comportement de leurs membres. Ce type d'enquêtes a connu une certaine stagnation à la fin du siècle et les descriptions de la vie des gangs étaient devenues un peu «plates », une absence de profondeur que l'on peut rattacher au manque d'intérêt des chercheurs pour l'aspect culturel à cette époque-là. Ces recherches montraient des gangs et des gangsters sans épaisseur.

69. Jones N. P., Understanding and addressing youth in gangs in Mexico, Retrieved at https://www. wilsoncenter. org/sites/default/files/jones_youth_gangs. pdf, 2013 ; Jones, G. A., " Hecho en México: Gangs, Identities and the Politics of Public Security ", in Hazen J. M., Rodgers D. (eds.), Global Gangs: Street Violence across the World, Mineápolis, University of Minnesota Press, 2014, pp. 255-280. 
La troisième période à laquelle nous avons fait référence dans cet article est la période contemporaine. Les travaux de cette époque commencent à la fin du XXe siècle et leur champ d'étude est principalement ouest-européen. C'est la finesse des descriptions qui rend ces travaux intéressants.

Enfin, la recherche ethnographique sur les gangs la plus récente se déroule en Amérique Latine, au Chili, au Mexique. Ces enquêtes sont récentes et ont souvent lieu dans des États faillis, ce qui place les sujets et les chercheurs dans une situation parfois dangereuse. Les travaux de Dennis Rodgers ont été une source d'inspiration méthodologique et théorique importante et ils reflètent la profondeur qui peut résulter de longues périodes de terrain et d'immersion dans une culture. Cette recherche a documenté l'évolution des gangs, allant de groupes locaux à des groupes plus organisés et plus violents prenant part à des activités de grande criminalité.

Nous conclurons cet article par quelques observations sur la période la plus récente des travaux d'ethnographie situés en Amérique Centrale et du Sud. De nombreuses recherches montrent qu'il y a une certaine intégration dans la relation entre les gangs et les populations locales, ce qui n'existe ni aux États-Unis, ni en Europe. Les gangs en Amérique Centrale et du Sud sont souvent des éléments importants de leurs communautés et ils sont souvent perçus comme des symboles de soutien et de stabilité, ce qui peut s'expliquer par l'incapacité des gouvernements de fournir la sureté, la sécurité ou la stabilité attendues par la population. Les groupes criminels de cette région ont connu des phases d'évolution importantes en fonction de leur degré d'intégration dans la société au niveau local et de leur niveau d'organisation. Les gangs sont par ailleurs très masculins, ce qui reflète la fonction de ces masculinités dans de nombreux pays de la région, en particulier auprès d'hommes marginalisés du point de vue économique et social. Ces groupes sont aussi touchés par le transnationalisme, et l'immigration et les exclusions ont suscité de nouveaux modes de comportement et d'affiliation. Il est clair que l'évolution du MS-13 au Salvador, au Guatemala et au Honduras prouve le rôle des modèles transnationaux de mobilité de population, qu'elle soit forcée ou volontaire. L'existence de nombreux petits États dans la région a également pu jouer un rôle dans ces mouvements de population, car les individus se déplacent d'un pays à un autre pour trouver un emploi, se mettre en sécurité, ou les deux. Enfin, l'économie politique de la région offre un autre moyen d'acquérir une position de pouvoir et de croître. De nombreux pays de la région ont peiné à maintenir une économie et un État stables, mais ils ont vu réduire leur capacité d'influence sur les comportements, en particulier ceux des jeunes marginalisés.

Les travaux ethnographiques antérieurs ont démontré l'utilité d'une telle approche. Il est d'ailleurs intéressant de noter que Dennis Rodgers lance une 
recherche globale sur les gangs, qui sera fondée sur les méthodes ethnographiques. Financé grâce à un European Research Grant, ce projet promet d'être une des études les plus ambitieuses jamais menée. Pour Rodgers, il y a encore trop peu de données collectées sur et avec les membres de gangs eux-mêmes 70 . Murray, Shenderovich, Gardner, Mikton, Derzon, Liu et Eisner ont produit une revue systématique de la recherche sur les facteurs de risque, qui a pu être menée dans des pays à revenus faibles à modérés, dans le cadre d'études longitudinales ${ }^{71}$. Cela témoigne de la faisabilité de l'utilisation d'instruments communs dans différents pays et auprès de différents groupes de populations. Le collectif de chercheurs autour de Katz mène une recherche en suivant le protocole de l'essai randomisé contrôlé (ERC - randomized control trial) du programme de prévention des gangs au Honduras, Proponte' Mas 72. Ce travail montre qu'il est possible de mettre en ouvre un plan de recherche sophistiqué dans la région. Par ailleurs, Decker et Pyrooz ont mené une enquête auprès de plus de deux-mille écoliers chinois ${ }^{73}$. Cela illustre la possibilité d'user des méthodes d'enquête dans des terrains que l'on croyait impénétrables. Ces exemples montrent que la méthodologie de recherche et de collecte de données préconisée par Rodgers et Baird est faisable et que de tels efforts pourraient venir compléter le nombre croissant et impressionnant des études ethnographiques dans la région. D'autres frontières demeurent, toutefois, car comme Decker et Pyrooz ont pu le souligner, dans de multiples cas, les véritables activités criminelles des gangs se déroulent en prison ${ }^{74}$. Si des travaux dans ce sens ont été conduits, il reste beaucoup à découvrir et l'accès aux prisons est encore rare et, quand il est possible, il est dangereux dans de nombreux cas ${ }^{75}$. L'avenir de la recherche sur les gangs engage des méthodes et des niveaux multiples; elle doit s'intéresser aux individus, aux groupes, aux espaces et à la variation dans le temps. Elle doit avoir un caractère transnational et inclure la participation du sujet au processus de recherche.

70. Rodgers D., Baird A., op cit., 2015.

71. Murray J., Shenderovich Y., Gardner F., Mikton C., Derzon Jianghong Liu J., Eisner M., "Risk Factors for Antisocial Behavior in Low-and Middle-Income Countries: A Systematic Review of Longitudinal Studies ", in Tonry M. (ed.), Crime and Justice, University of Chicago Press, 2018.

72. Secondary Violence Prevention Activity, Proponte’ Mas, USAID, 2016.

73. Pyrooz D., Decker S., "Delinquent Behavior, Violence and Gang Involvement in China ", Journal of Quantitative Criminology, 29, 2013, pp. 251-272.

74. Decker S., Pyrooz D., op cit., 2016.

75. Cunha M., "The Ethnography of Prisons and Penal Confinement ", Annual Review of Anthropology, 43, 2014, pp. 217-233 ; Dias C., Salla F., op cit., 2013. 